\title{
AÇÕES EDUCATIVAS PARA PROMOÇÃO DA SAÚDE NA ESCOLA: REVISÃO INTEGRATIVA
}

Lia Maristela da Silva Jacob

Doutora em Ciências da Saúde pela Universidade Estadual de Campinas - UNICAMP, Brasil

\section{Márcio Cristiano de Melo}

Doutorando em Saúde Coletiva pela Universidade Estadual de Campinas - UNICAMP, Brasil

\section{Rômulo Mágnus de Castro Sena}

Doutorando em Ciências Médicas/Saúde Mental pela Universidade Estadual de Campinas - UNICAMP, Brasil

\section{Isaac Jacob da Silva}

Especialista em Fisioterapia Traumato-Ortopédica Funcional pela Universidade de Fortaleza - UNIFOR, Brasil.

\section{Reginaldo Roque Mafetoni}

Doutor em Ciências da Saúde pela Universidade Estadual de Campinas - UNICAMP, Brasil

\section{Kellen Cristina Silva de Souza}

Docente no curso de Graduação em Pedagogia pela Faculdade Unitelos, Brasil.
RESUMO: Identificar e descrever as evidências científicas sobre ações educativas realizadas pelo Programa Saúde na Escola. Revisão integrativa, com levantamento bibliográfico entre os anos 2014 e 2018, em bases de dados eletrônicas com tradição em publicações na área da saúde. O levantamento resultou em 12 artigos que abordaram os seguintes eixos: estudo cartográfica na escola, uso de web rádio com escolares, conhecimento de escolares sobre poluição, percepções de educadores sobre educação inclusiva, ações de alimentação e nutrição na escola, enfermeiros no contexto escolar, promoção da saúde auditiva, saúde em disfonia infantil, atenção primária à saúde de escolares, práticas intersetoriais e acidentes de trabalho entre escolares. A educação em saúde é apontada como importante estratégia para alcance de indicadores positivos, no que diz respeito à promoção da saúde e prevenção de doenças entre escolares.

PALAVRAS-CHAVE: Serviços de Saúde Escolar; Promoção da saúde; Educação em saúde.

\section{EDUCATIONAL ACTIVITIES FOR HEALTH PROMOTION IN SCHOOL: INTEGRATIVE REVIEW}

ABSTRACT: To identify and describe scientific evidences on educational activities undertaken by the School Health Program. Integrative review, coupled to bibliographical survey, between 2014 and 2018, based on electronic data with regard to publication in health. Survey pinpointed twelve articles on the following items: cartographic study of the school, the use of web radio with students, students' knowledge on pollution, perception of educators with regard to inclusive education, feeding and nutrition in school, nursing within the school context, audition health promotion, children's dysphonia, first aid to students, intersecting practices and labor accidents among students. Health education is the main strategy to attend to positive indexes with regard to health promotion and prevention of diseases among students.

KEY WORDS: School health service; Health promotion; Health education.

\section{INTRODUÇÃO}

Autor correspondente

Lia Maristela

lia_maristela@hotmail.com
A promoção da saúde é definida, atualmente, como conjunto de estratégias e formas de produzir saúde, no âmbito individual e coletivo, caracterizando-se pela articulação e cooperação intra e inter- 
setorial, a qual busca articular ações com as demais redes de proteção social, com participação e controle social" 1. Para tanto, foi criado, em 2007, o Programa Saúde na Escola (PSE), prospecto institucional que se instalou com base no escopo da promoção da saúde, em articulação com a Estratégia Saúde da Família. Propõe-se a integrar e articular os dois setores com ações que objetivam melhorar a qualidade de vida de alunos de escolas de educação básica.

O programa é desenvolvido com ênfase em cinco componentes: adolescentes e jovens das escolas públicas; avaliação das condições de saúde das crianças; educação continuada e capacitação dos profissionais da educação e da saúde e de jovens; monitoramento e avaliação da saúde dos estudantes; monitoramento; avaliação do programa e promoção da saúde e ações de prevenção de doenças e agravos à saúde 2 .

Destaca-se que a contribuição da intersetorialidade frente às ações de PS, pois experiências promissoras, como as Escolas Promotoras da Saúde, trouxeram novas abordagens, priorizando a promoção da saúde e a intersetorialidade, todavia, na prática, os avanços foram limitados e incapazes de gerar ações integradas³.

Atualmente, a temática saúde na escola recebe importante atenção de organismos internacionais, em especial, a Organização Mundial da Saúde (OMS) e a Unesco, o que confirma a relevância do assunto em âmbito mundial ${ }^{4}$. Sobretudo, por se tratar de intervenções em fase de desenvolvimento importante na vida do escolar, rumo à adolescência, etapa valiosa na aquisição de conhecimentos e adoção de atitudes e comportamentos protetivos sobre saúde, com repercussões para qualidade de vida futura ${ }^{5}$.

Face ao exposto, as ações de educação em saúde exigem compreensão ampliada de cuidado de saúde, sendo necessária a participação de usuários na mobilização, capacitação e no desenvolvimento de aprendizagem de habilidades individuais e sociais para lidar com os processos de saúde-doença ${ }^{6}$.

Estudo aponta que a educação em saúde, enquanto processo, pensar, fazer pedagógico emancipatório, ou seja, na possibilidade do desenvolvimento da autonomia intelectual dos sujeitos, individual e coletiva, envolve maior aproximação com o adolescente, visto que devem ser consideradas as particularidades de cada grupo, bem como o entorno social em que estão inseridos ${ }^{8}$.

Nesse contexto, objetivou-se identificar e descrever as evidências científicas sobre as ações educativas realizadas no Programa Saúde na Escola.

\section{METODOLOGIA}

Para obtenção de síntese dos resultados de pesquisa relevantes e reconhecidos nacionalmente, utilizou-se como método a revisão integrativa ${ }^{8}$.

Partiu-se da pergunta norteadora: quais as ações educativas realizadas no Programa Saúde na Escola frente à promoção da saúde? A pesquisa foi realizada entre julho e agosto de 2018, por meio de consulta às bases bibliográficas eletrônicas: Medical Literature Analysis and Retrieval System Online (MEDLINE) via Pubmed, Literatura Latino-americana e do Caribe em Ciências da Saúde (LILACS), Banco de Dados em Enfermagem (BDENF), Acervo da Biblioteca da Organização Pan-Americana da Saúde (PAHO), Sistema de Informação da Biblioteca da OMS (WHOLIS), Literatura do Caribe em Ciências da Saúde (MEDCARIB) via Portal Regional da Biblioteca Virtual em Saúde (BIREME) e Scientific Electronic Library Online (SciELO), no período de 2014 a 2018.

Os termos de busca foram obtidos a partir dos Descritores em Ciências da Saúde (DeCS), em português e inglês. Foram utilizados os sinônimos "Saúde Escolar" e "Programa Saúde na Escola" do descritor "Serviços de Saúde Escolar", "Promoção da Saúde" e "Educação em Saúde". Utilizou-se a expressão booleana "AND", cruzando-se os sinônimos do primeiro descritor (e o próprio) com o segundo ou o terceiro: "Saúde Escolar" AND "Promoção da Saúde"; "Saúde Escolar" AND "Educação em Saúde"; "Programa Saúde na Escola" AND "Promoção da Saúde"; "Programa Saúde na Escola" AND "Educação em Saúde"; "Serviços de Saúde Escolar" AND "Promoção da Saúde"; "Serviços de Saúde Escolar" AND "Educação em Saúde". Especificamente nas bases MEDLINE e PubMed, foram utilizados os MeSH Terms: "School Health Services", "School Health Services" AND "Health Promotion" e "School Health Services" AND "Health Education".

Nas bases de dados selecionadas, realizou-se 
pesquisa em português, por se tratar de bases de dados que apresentam estudos relevantes que retratam o Programa Saúde na Escola brasileiro.

Como critérios de inclusão, consideraram-se: artigos na íntegra, disponíveis on-line, publicados em português, que tratassem do tema da pesquisa e respondessem à pergunta norteadora. Os critérios de exclusão foram os artigos incompletos, com acesso apenas ao resumo, os artigos que tratassem de ações educativas em saúde como qualquer outro tema (atenção primária, cuidado continuado etc.), as teses e dissertações, a carta ao editor, e que não respondiam à pergunta norteadora.

Títulos e resumos dos 113 artigos encontrados, após exclusão das duplicações, foram lidos por dois avaliadores separadamente e, caso estivessem no tema da revisão, eram lidos na íntegra, sendo preenchida tabela com dados dos artigos que responderam à pergunta norteadora. Após leitura dupla dos artigos, caso houvesse discordância quanto à inclusão ou exclusão, este foi lido por terceiro avaliador para decisão sobre inclusão ou não. O fluxograma da seleção dos artigos que compuseram a revisão integrativa encontra-se na Figura 1.

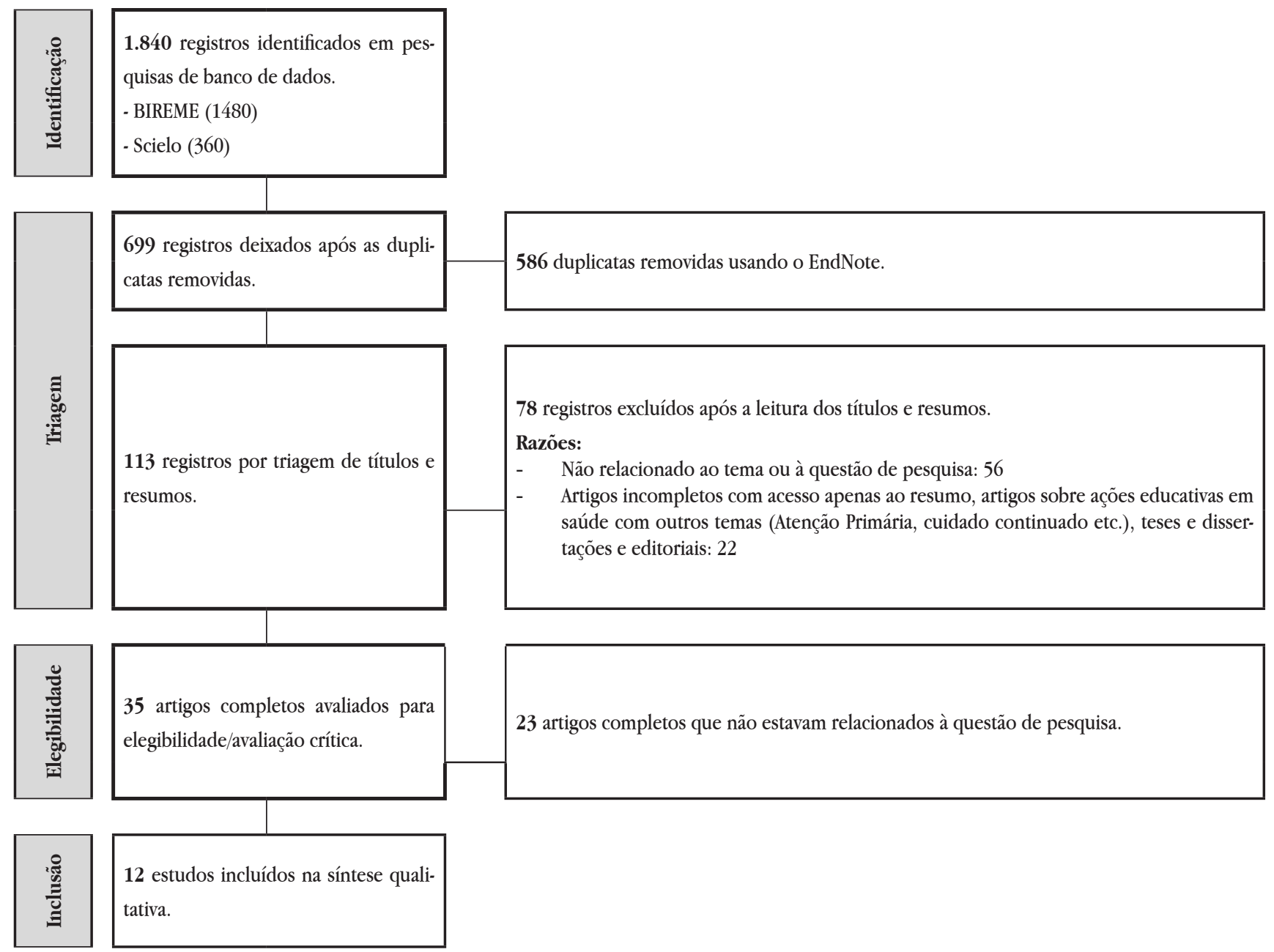

Figura 1. Fluxograma da PRISMA de seleção dos estudos que compuseram a revisão integrativa, 2014 a 2018. Fonte: Elaborada pelos autores. 


\section{RESULTADOS}

Foram incluídos 12 artigos nesta revisão, os quais se encontram descritos no Quadro1, organizados por ano de publicação.

Em relação ao ano de publicação, encontraram-se cinco artigos em 2015 e 2016, respectivamente, um artigo em 2014, e um em 2017.

A análise do conjunto de artigos permitiu a construção de quatro categorias temáticas, com base no objetivo deste estudo. O critério de categorização obedeceu à temática principal abordada. Logo, as fontes que apresentaram o mesmo contexto ou ideia foram agrupadas segundo as quatro categorias: 1) saúde fonoaudiológica; 2) meio ambiente e saúde; 3) saúde nutricional; e 4) metodologias e materiais didáticos na educação em saúde.

Quadro 1. Caracterização dos estudos segundo autoria, ano de publicação, delineamento do estudo e principais achados, 2014 a 2018

\begin{tabular}{|c|c|c|}
\hline Artigos & Delineamento metodológico & Principais achados \\
\hline França e Lacerda, $2014^{9}$ & $\begin{array}{l}\text { Relato de experiência, utilizando pressupostos da pedagogia proble- } \\
\text { matizadora, realizado com os alunos do ensino médio de uma escola } \\
\text { estadual, em Santa Catarina, sobre os efeitos do ruído na saúde. }\end{array}$ & $\begin{array}{l}\text { Os resultados reforçam a importância da implemen- } \\
\text { tação de programas de promoção de saúde auditiva } \\
\text { para adolescentes, com foco no envolvimento dos es- } \\
\text { colares e valorização da saúde auditiva na qualidade } \\
\text { de vida estudantil. }\end{array}$ \\
\hline $\begin{array}{l}\text { Dias, Cruz e Carvalho, } \\
2015^{10}\end{array}$ & $\begin{array}{l}\text { Elaboração de material didático de educação para a saúde em forma de } \\
\text { livro de literatura infantil para terapia indireta sobre disfonia infantil. }\end{array}$ & $\begin{array}{l}\text { Destacou-se a dupla possibilidade de utilização do } \\
\text { material: para fins no setting terapêutico e no con- } \\
\text { texto cotidiano da criança ultrapassando a barreira } \\
\text { clínica e permitindo o trabalho terapêutico na vida } \\
\text { real da criança, permitindo o envolvimento dos pais } \\
\text { e educadores/professores no processo de reeducação } \\
\text { vocal. }\end{array}$ \\
\hline $\begin{array}{l}\text { Gomes, Câmara e Sou- } \\
\text { za, } 2015^{11}\end{array}$ & $\begin{array}{l}\text { Estudo transversal sobre o conhecimento prévio e posterior dos esco- } \\
\text { lares sobre poluição e os efeitos à saúde provocados por um ambiente } \\
\text { impactado pela destinação inadequada dos resíduos sólidos. }\end{array}$ & $\begin{array}{l}\text { As atividades dos escolares, com a aquisição de novos } \\
\text { conhecimentos, proporcionaram uma reflexão crítica } \\
\text { sobre o ambiente e como este pode impactar a saúde } \\
\text { humana. }\end{array}$ \\
\hline $\begin{array}{l}\text { Salum e Monteiro, } \\
2015^{12}\end{array}$ & $\begin{array}{l}\text { Relato de experiência de um grupo de discentes do } 9^{\circ} \text { período de En- } \\
\text { fermagem de um centro universitário privado de Sete Lagoas-MG, acer- } \\
\text { ca de uma intervenção em educação em saúde do adolescente. }\end{array}$ & $\begin{array}{l}\text { A prática educativa com adolescentes possibilitou ao } \\
\text { grupo a aproximação com estudantes adolescentes } \\
\text { de uma instituição pública de ensino em um contex- } \\
\text { to social de vulnerabilidades, sendo possível destacar } \\
\text { a escola como um locus estratégico para ações em } \\
\text { saúde dessa natureza. }\end{array}$ \\
\hline $\begin{array}{l}\text { Souza, Lira, Andrade, } \\
\text { Cabral, Carvalho e Oli- } \\
\text { veira, } 2015^{13}\end{array}$ & $\begin{array}{l}\text { Estudo qualitativo realizado com entrevista semiestruturada com nu- } \\
\text { tricionistas de sete municípios do Estado de Pernambuco sobre ali- } \\
\text { mentação adequada e saudável no ambiente escolar, na perspectiva do } \\
\text { Programa Saúde na Escola. }\end{array}$ & $\begin{array}{l}\text { Ressalta-se que a consolidação do nutricionista se faz } \\
\text { importante para resolução de problemas voltados } \\
\text { para alimentação e nutrição através da qualificação } \\
\text { continuada desse profissional, com ênfase nas ações } \\
\text { de promoção da alimentação saudável e adequada e } \\
\text { respeito à diversidade e cultura local. }\end{array}$ \\
\hline $\begin{array}{l}\text { Torres, Silva, Bezerra, } \\
\text { Abreu e Mendonça, } \\
2015^{14}\end{array}$ & $\begin{array}{l}\text { Relato de experiência de acadêmicos de Enfermagem da Universidade } \\
\text { Estadual Vale do Acaraú, em uma escola de educação infantil e ensi- } \\
\text { no fundamental no Ceará, com os jovens escolares que participaram } \\
\text { do Programa em Sintonia com a Saúde, postado ao vivo por uma Web } \\
\text { Rádio. }\end{array}$ & $\begin{array}{l}\text { Verificou-se que a utilização da ferramenta tecnoló- } \\
\text { gica no desenvolvimento do processo educativo pro- } \\
\text { posto que superou o modelo tradicional para o foco } \\
\text { da coprodução de saberes e autonomia dos jovens. }\end{array}$ \\
\hline
\end{tabular}




\begin{tabular}{|c|c|c|}
\hline Artigos & Delineamento metodológico & Principais achados \\
\hline $\begin{array}{l}\text { Farias, Sá, Figueiredo e } \\
\text { Menezes Filho, } 2016^{15}\end{array}$ & $\begin{array}{l}\text { Estudo de caso com problematização dos limites e potencialidades no } \\
\text { processo de construção da intersetorialidade a partir da compreensão } \\
\text { do Programa Saúde na Escola (PSE), no Estado de Pernambuco. }\end{array}$ & $\begin{array}{l}\text { A implantação do PSE permitiu aos profissionais de } \\
\text { saúde a percepção do seu papel social de educador } \\
\text { e possibilitou aos adolescentes maior contato com a } \\
\text { equipe de saúde. Houve aproximação entre escola e } \\
\text { unidade de saúde contribuindo para ajudar os ado- } \\
\text { lescentes a transformar a informação científica em } \\
\text { comportamentos saudáveis. }\end{array}$ \\
\hline Melo e Barros, 2016 & $\begin{array}{l}\text { Pesquisa-Intervenção com método cartográfico com base nas atuações } \\
\text { de estudantes no estágio profissionalizante em psicologia e saúde co- } \\
\text { letiva, no campo da Atenção Primária e Saúde, abordando as práticas } \\
\text { de saúde desenvolvidas por profissionais de saúde e educação no co- } \\
\text { tidiano da escola. }\end{array}$ & $\begin{array}{l}\text { Na escola, as práticas de saúde estão voltadas em sua } \\
\text { maioria a questões de alimentação saudável e higie- } \\
\text { nização do corpo. A cartografia permitiu acompanhar } \\
\text { e produzir processos e fluxos, como no caso da roda } \\
\text { de conversa sobre saúde, de problematização das } \\
\text { práticas instituídas, experimentando, por conseguin- } \\
\text { te, olhares voltados à desindividualização da saúde } \\
\text { do aluno, afirmando-a como responsabilidade de } \\
\text { todos. }\end{array}$ \\
\hline $\begin{array}{l}\text { Silva, Molero e Roman, } \\
2016^{17}\end{array}$ & $\begin{array}{l}\text { Entrevistas semiestruturadas com professoras de ensino fundamental } \\
\text { de escolas municipais em Santos-SP com foco nas dimensões relacio- } \\
\text { nadas à educação inclusiva, dentre elas as inter-relações entre as áreas } \\
\text { da saúde e educação. }\end{array}$ & $\begin{array}{l}\text { Apesar dos avanços nos processos de educação inclu- } \\
\text { siva, observa-se ainda uma inclusão que busca se ade- } \\
\text { quar aos moldes legislativos, definidos pelos profes- } \\
\text { sores como "lindo e perfeito no papel", mas que, na } \\
\text { prática, tem se apresentando de modo fragmentado } \\
\text { em diferentes instâncias da atenção à criança com de- } \\
\text { ficiência e/ou transtorno global do desenvolvimento. }\end{array}$ \\
\hline Silva e Bodstein, $2016^{3}$ & $\begin{array}{l}\text { Estudo sobre os referenciais teóricos que influenciaram os programas } \\
\text { de saúde escolar no Brasil. Realizou-se recuperação e identificação de } \\
\text { documentos oficiais, principalmente de publicações referentes à saúde } \\
\text { escolar, selecionadas desde a década de } 20 \text { até } 2009 \text {, na cidade do Rio } \\
\text { de Janeiro. }\end{array}$ & $\begin{array}{l}\text { O desenvolvimento histórico dessa articulação in- } \\
\text { tersetorial, tendo como cenário a escola, revelou } \\
\text { precariedade das articulações e fragilidade do diálo- } \\
\text { go intersetorial. O discurso biomédico hegemônico } \\
\text { enuncia questóes prioritárias sob a ótica da saúde, } \\
\text { muito pouco problematizadas pela comunidade es- } \\
\text { colar nos contextos históricos aqui analisados. }\end{array}$ \\
\hline $\begin{array}{l}\text { Gomes, Câmara e Sou- } \\
\text { za, } 2016^{18}\end{array}$ & $\begin{array}{l}\text { Estudo transversal que incluiu a aplicação de um mesmo questionário } \\
\text { entre alunos matriculados numa escola da rede municipal de Cuiabá- } \\
\text {-MT, Brasil, antes e depois de atividades de educação para a saúde vol- } \\
\text { tadas para a definição dos acidentes de trabalho. }\end{array}$ & $\begin{array}{l}\text { Os resultados obtidos na segunda fase do programa } \\
\text { educativo aplicado aos escolares de uma escola im- } \\
\text { pactada por aterro sanitário evidenciou, de forma } \\
\text { positiva, a modificação do conhecimento entre os } \\
\text { participantes sobre acidentes de trabalho. }\end{array}$ \\
\hline Folha e Monteiro, $2017^{19}$ & $\begin{array}{l}\text { Estudo qualitativo do tipo intervenção com alunos que apresentavam } \\
\text { queixas escolares e os professores que acompanhavam esses casos em } \\
\text { uma Unidade de Educação Infantil em Belém-PA. Foi realizada obser- } \\
\text { vação direta do desempenho dos alunos e intervenção ancorada na } \\
\text { abordagem de consultoria colaborativa. }\end{array}$ & $\begin{array}{l}\text { A parceria entre terapeuta ocupacional e escola, por } \\
\text { meio da consultoria colaborativa, potencializou pro- } \\
\text { cessos educacionais inclusivos, no sentido da promo- } \\
\text { ção e prevenção de agravos à saúde do escolar. }\end{array}$ \\
\hline
\end{tabular}




\section{DISCUSSÃO}

A maioria das publicações se concentrou nos anos de 2015 e 2016. Em relação à pergunta de pesquisa, as publicações demonstraram avanços nas ações voltadas ao Programa Saúde na Escola, fortalecendo as políticas de inclusão da assistência à saúde, respeitando os cenários de vivência das pessoas.

A intensificação das publicações a respeito da temática, a partir de 2015, também se evidencia em países como Portugal que implementou o Programa Nacional de Saúde Escolar (PNSE), enquanto instrumento orientador das políticas nacionais de saúde e a participação de outros setores ${ }^{20}$. Tal programa visa o fortalecimento das capacitações bilaterais dos profissionais, estilos de vida, ambiente escolar saudável com promoção do desempenho escolar, bem como qualidade da formação, de inovações dos métodos e de investigações.

Nessa perspectiva, é interessante que profissionais de saúde tenham, em formação acadêmica, vivência no âmbito escolar, facilitando, assim, o processo de aprendizagem e a atuação profissional durante a comunicação com estudantes.

\section{SAÚDE FONOAUDIOLÓGICA}

Ao enfocar a educação infantil, a criação de tecnologia leve com abordagem lúdica é muito eficaz para desenvolver ação de educação em saúde nesta faixa etária. Dados apresentados no estudo de Dias, Cruz e Carvalho $(2015)^{10}$, concebido sob a forma de livro de fábulas, emergem do contexto da criação de instrumentos lúdico-pedagógicos destinados à reestruturação das ferramentas clínicas na comunicação relacional e pedagógica entre o fonoaudiólogo e a criança.

Estudo realizado em Santa Catarina, Brasil, apontou a implementação de programas de promoção da saúde auditiva para escolares que contemplem o desenvolvimento de ações de sensibilização ao risco e à importância da saúde auditiva na qualidade de vida. A presença constante do fonoaudiólogo e demais profissionais envolvidos com a temática seria benéfica e poderia contribuir para promoção da saúde auditiva de escolares de forma efetiva e permanente?.

\section{MEIO AMBIENTE E SAÚDE}

Pesquisa aponta que as atividades dos escolares, com aquisição de novos conhecimentos, direcionam reflexão crítica sobre o ambiente e como este pode interferir na saúde humana. Percebeu-se que é significante respeitar o saber acumulado dos sujeitos para seguir nova discussão, que integre saberes existentes com os obtidos por meio da atividade de intervenção ${ }^{11}$.

No entanto, contextualiza-se a educação com saúde ocupacional e se percebe que admirar o saber de escolares, considerando o conhecimento prévio sobre questões ocupacionais, e fomentar a busca por novos saberes dentro do contexto, pode ser guia condutor para construção de novas práticas, visando prevenção de doenças e agravos à saúde ${ }^{18}$.

\section{SAÚDE NUTRICIONAL}

Pesquisa qualitativa, realizada na região Nordeste do Brasil, identificou que a consolidação do nutricionista na Atenção Básica à Saúde se faz importante para resolução de problemas voltados para alimentação e nutrição na escola, tendo em vista a garantia da segurança alimentar e nutricional. No entanto, faz-se necessária a qualificação continuada desse profissional, com ênfase nas ações de promoção da alimentação saudável e adequada e respeito à diversidade e cultura local ${ }^{13}$.

\section{METODOLOGIAS E MATERIAIS DIDÁTICOS NA EDUCA- ÇÃO EM SAÚDE}

A literatura aponta que o Programa Saúde na Escola, embora tenha sido desenvolvido em 2007, ainda não é amplamente utilizado, em razão de entraves do cotidiano dos profissionais. Existe busca de consolidação de práticas intersetoriais que, na maioria das vezes, são substituídas por ações pontuais. Portanto, pode-se pensar em intervenções mais concretas para fortalecer a intersetorialidade e as ações intersetoriais no PSE $^{15}$.

Desse modo, a ação intersetorial deve ser negociada e incluída na rotina e prática dos profissionais, permitindo construção de conhecimentos mais dialógicos e contextualizados para políticas de saúde mais efetivas na escola ${ }^{3}$. 
Pesquisa realizada no Piauí, Brasil, constatou que frente à visão dos profissionais de saúde acerca das práticas de saúde na escola, estas assumem caráter de vigilância epidemiológica, reduzindo-se, muitas vezes, à descrição das condições de saúde dos alunos. Portanto, possibilitou a desnaturalização das concepções de saúde, cujas práticas podem ir além da realização de atividades voltadas às práticas normativas, como higiene e alimentação, pensando-se em outros modos de produzir saúde ${ }^{16}$.

Esse pensamento vem de encontro com estudo realizado durante a formação dos acadêmicos de enfermagem, em que estudantes desenvolveram ferramenta tecnológica, permitindo que estes se inserirem em novos arranjos para implementação de ações com vistas à promoção da saúde, em dimensão mais dialógica, interativa e rica de novos saberes, através dos entornos da vida cotidiana de jovens na escola e comunidade ${ }^{14}$.

Face ao exposto, a ação educativa em saúde na escola para adolescente viabilizou aos acadêmicos de enfermagem novos métodos de agir em saúde na atenção primária, tendo na escola cenário favorável ao exercício da educação em saúde, como interface do cuidado de enfermagem na atenção primária à saúde ${ }^{12}$.

Ao considerar que a escola é local propício para promoção da saúde, Folha e Monteiro (2016) ${ }^{19}$ concluíram que a intervenção na atenção primária em saúde possibilita ao profissional o desenvolvimento de ações que abrangem promoção e proteção da saúde, redução de danos, bem como manutenção da saúde, prevenção de agravos, aspectos que estão na essência da prática do terapeuta ocupacional.

Em relação à educação inclusiva, pesquisadores sinalizam que entre as estratégias de intervenção, certamente, estão a necessidade de reformulação da formação de profissionais de saúde que precisam compreender os problemas e as demandas escolares, a articulação entre instituições acadêmicas e redes de serviços para desenvolvimento de pesquisas, métodos e formação contínua, e reestruturação do funcionamento escolar, a fim de valorizar professores e alunos e, assim, oferecer melhores condições para o fazer educativo, de modo a promover o desenvolvimento de todos ${ }^{17}$.

\section{CONSIDERAÇÕES FINAIS}

De acordo com os estudos revisados, a educação em saúde vem sendo apontada como uma das melhores estratégias para alcance de indicadores positivos, no que diz respeito à promoção da saúde e prevenção de doenças em escolas.

Ademais, a intersetorialidade entre saúde e educação vem sendo apontada por pesquisadores de ambas as áreas. Deste modo, profissionais conseguem desenvolver metodologias relevantes e direcionar abordagem significativa a usuários de instituições de saúde e ensino.

É importante que profissionais de saúde e educadores escolares se fortaleçam frente às ações direcionadas à comunidade, pois políticas públicas, ainda, precisam ser discutidas e implementadas coletivamente, viabilizando dados epidemiológicos significativos na prevenção de agravos à saúde.

\section{REFERÊNCIAS}

1. Ministério da Saúde (BR). Portaria n ${ }^{\circ} 2.446$, de 11 de novembro de 2014: Redefine a Política Nacional de Promoção da Saúde (PNPS). Brasília (DF): Ministério da Saúde; 2014.

2. Ministério da Saúde (BR). Secretaria de Atenção à Saúde. Departamento de Atenção Básica. Ministério da Educação. Passo a Passo PSE. Programa Saúde na Escola: tecendo caminhos da intersetorialidade. Brasília (DF): Ministério da Saúde; 2011.

3. Silva CS; Bodstein RCA. Referencial teórico sobre práticas intersetoriais em Promoção da Saúde na Escola. Ciênc. saúde coletiva. [Internet] 2016 Jun [acesso 2018 Nov 29]; 21(6): 1777-88. Disponível em: http://www.scielo.br/pdf/csc/v21n6/1413-8123csc-21-06-1777.pdf

4. Carvalho FFB. A saúde vai à escola: a promoção da saúde em práticas pedagógicas. Physis. [Internet] 2015 Dez [acesso 2018 Nov 29]; 25(4): 1207-27. Disponível em: http://www.scielo.br/scielo.php?script $=$ sci arttext\&pid $=$ S0103-73312015000401207\&lng $=$ en. Doi: $\quad$ http://dx.doi.org/10.1590/S010373312015000400009 .

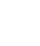


5. Marin C, Papadopol PM, Bottan ER, Orcina BF. Percepção E Informação Sobre Saúde Bucal: Estudo Com Adolescentes De Uma Escola Pública.Rev. saúde pesq. [Internet] 2016 Set/Dez [acesso 2018 Nov 29]; 9(3): 499-506. Disponível em: http://periodicos.unicesumar.edu.br/index.php/saudpesq/article/ view/5417/2917.

6. Machado MFAS, Vieira NFC. Educação em saúde: o olhar da equipe de saúde da família e a participação do usuário. Rev. Latino-Am. Enfermagem. [Internet]. $2009 \mathrm{Abr}$ [acesso 2018 Nov 29]; 17(2): 174-179. Disponível em: http:// www.scielo.br/scielo.php?script $=$ sci_arttext\&pi$\mathrm{d}=$ S0104-11692009000200006\&lng=en. Doi: http:// dx.doi.org/10.1590/\$0104-11692009000200006.

7. Leite CT, Vieira RP, Machado CA, Quirino GS, Machado MFAS. Prática de educação em saúde percebida por escolares. CogitareEnferm. [Internet]. 2014 Jan/Mar [acesso 2018 Nov 29]; 19(1):13-19. Disponível em: https://revistas.ufpr.br/cogitare/article/ view/35925/22157.

8. Mendes KDS, Silveira RCDCP, Galvão CM. Revisão integrativa: método de pesquisa para a incorporação de evidências na saúde e na enfermagem. Texto Contexto Enferm, Florianópolis, 2008 Out-Dez; 17(4): 758-64.

9. França $A G$, Lacerda ABM. Promoção da saúde auditiva: estratégias educativas desenvolvidas por estudantes do ensino médio. DistúrbComun, São Paulo, 2014 Mar; 26(1): 365-72.

10. Dias MR, Cruz CV, Carvalho AR. "Barnabé e sua aventura": um projeto de educação para saúde em disfonia infantil. Distúrb Comum, São Paulo, 2015; 27(2): 293-300.

11. Gomes RMV, Câmara VM, Souza DPO. Escolares residentes em área impactada por aterro sanitário e seu conhecimento sobre poluição. Cad. Saúde Colet., Rio de Janeiro, 2015; 23 (4): 445-52.

12. Salum GB, Monteiro LAS. Educação em saúde para adolescentes na escola: um relato de experiência. Rev. mineira enferm., 2015; 19(2): 246-57. lho AT, Oliveira JS. O Programa Saúde na Escola e as ações de alimentação e nutrição: uma análise exploratória. Rev. APS, 2015 jul/set; 18(3): 360-7.

14. Torres RAM, Silva MAM, Bezerra AEM, Abreu LDP, Mendonça GMM. Comunicação em saúde: uso de uma web rádio com escolares. J. Health Inform., $2015 \mathrm{Abr} / J u n ; 7(2): 58-61$.

15. Farias ICV, Sá RMPF, Figueiredo N, Menezes Filho A. Análise da intersetorialidade no Programa Saúde na escola. Rev. bras. educ. med., 2016; 40(2): 261-7.

16. Melo ACV, Barros JPP. Práticas de saúde na escola: um estudo cartográfico na cidade de Parnaíba-PI. Pesquisas e Práticas Psicossociais, 2016; 11(2): 341-55.

17. Silva CCB, Molero ESS, Roman MD. A interface entre saúde e educação: percepções de educadores sobre educação inclusiva. Psicologia Escolar e Educacional, $2016 \mathrm{Jan} / \mathrm{Abr} ; 20(1)$ : 109-15.

18. Gomes RMV, Câmara VM, Souza DP. Modificação do conhecimento sobre acidentes de trabalho entre escolares residentes de uma área impactada por aterro sanitário. Rev. bras. epidemiol. [Internet]. 2016 Set [acesso 2018 Dez 01]; 19(3): 632-44. Disponível em: http://www.scielo.br/scielo.php?script =sci_arttext\&pid $=\$ 1415-790 X 2016000300632 \& \operatorname{lng}=$ en. Doi: http://dx.doi.org/10.1590/1980-5497201600030013.

19. Folha DRSC, Monteiro GS. Terapia ocupacional na atenção primária à saúde do escolar visando a inclusão escolar de crianças com dificuldades de aprendizagem. Revista Interinstitucional Brasileira de Terapia Ocupacional, 2017; 1(2): 202-20.

20. Ministério da Saúde (PT). Direção-Geral da Saúde. Programa Nacional de Saúde Escolar. Lisboa: DGS; 2015. $105 \mathrm{p}$. 